\title{
Comparative Study of Anti-Mullerian Hormone, inhibin-B, and Three- Dimensional Ultrasound Determinants of Ovarian Reserve in Patients Undergoing ICSI
}

\author{
Farid I. Hassan, Walid A. El Sheikh, Wael R. Hablas, Doaa E. Abou Shama \\ *Obstetrics and Gynecology Department, Faculty of Medicine, Al-Azher University- Cairo Egypt \\ Corresponding auther: Doaa elsayed Abou Shama, E mail: doaaaboushama @ gmail .com
}

\begin{abstract}
:
Background: Intracytoplasmic sperm injection (ICSI) is an in vitro fertilization procedure in which a single sperm is injected into an oocyte. Objective: Aim of the Work: Comparing threedimensional ultrasound parameters, with anti-Mullerian hormone $(\mathrm{AMH})$ and other conventional endocrine markers for the prediction of ovarian response in patients undergoing intracytoplasmic sperm injection cycles. Methods: This observational prospective study was conducted at Alexandria Armed Military Hospital, on a sample of 100 women aged between 25 and 35 years, enrolled for intracytoplasmic sperm injection (ICSI). Results: a good correlation between inhibin B and other numerical variables. There was strong positive correlation between inhibin B and AMH, strong negative correlation between inhibin B and both FSH and LH. Inhibin B correlated moderately negatively with age and moderately positively with AFC, number of collected oocytes. Inhibin B correlated positively with the number of oocytes and number of grade 1 embryos. As regard pregnancy rate, the good-response group had significantly higher chemical and clinical pregnancy rates, as there was no chemical or clinical pregnancy occurred in poor responders. Conclusion: AFC, $\mathrm{AMH}$ and Inhibin B are effective in predicting the ovarian reserve as well as the response to induction, and both AFC and AMH are the most significant predictors of response to ovarian stimulation during ICSI. Recommendations: Larger scale studies including higher number of patients are needed to verify the validity of this parameter as a marker of ovarian reserve in patients undergoing ICSI.
\end{abstract}

Key words: anti-Mullerian hormone, inhibin B, three dimensional ultrasound, ovarian reserve, ICSI.

\section{INTRODUCTION}

Infertility is a disease of the reproductive system defined as the failure to achieve a clinical pregnancy after 12 months or more of regular unprotected intercourse ${ }^{(1)}$. Since the stabilization of in vitro fertilization, it became obvious that almost half of the couples failed to realize fertilization and that phenomenon was assign to a male gamete dysfunction. The adoption of assisted fertilization techniques especially ICSI has been able to mitigate male factor infertility by giving the consistent ability of a viable spermatozoon to activate an oocyte (2). Intracytoplasmic sperm injection (ICSI) is an in vitro fertilization procedure in which a single sperm is injected directly into an oocyte. This procedure is most ordinarily used to cope male infertility problems, though it may also be used where eggs cannot easily be permeated by sperm and sometimes sperm donation ${ }^{(3)}$.

A number of predictors intracytoplasmic sperm Injection (ICSI) have been identified, including patient age; concentration of hormonal markers, such as basal FSH, LH, estradiol, inhibin B, and of ovarian response to, more recently, $\mathrm{AMH}$; and dynamic tests, such as the clomiphene citrate challenge test ${ }^{(4)}$

Other predictors of ovarian reserve include ultrasound measures, such as pre-treatment ovarian volume and AFC, which is the total of small antral follicles in both ovaries. Generality of these measures, however, have restricted predictive value, oftentimes because in fact predictors of ovarian reserve not ovarian response, e.g. AMH, or have fundamental inter-patient variability, e.g. age, or intra-cycle fluctuation, e.g. basal FSH ${ }^{(5)}$. Patients with very low ovarian reserve may still become pregnant, and those with high ovarian reserve might not attain ongoing pregnancy. Nevertheless, previous studies has been tailored established on markers of ovarian response offers that outcomes are amended and the incidence of low and high response reduced compared with standard ICSI treatment ${ }^{(6)}$.

Since serum AMH levels be inverted the ovarian follicular pool, any lowering in serum $\mathrm{AMH}$ level denotes reduction in the number of small growing follicles. So AMH is a preferable marker and is superior to other markers ${ }^{(7)}$.

Anti-mullerian hormone is generated by 
pre-antral follicles. The highest level of AMH expression is existing in granulosa cells of secondary, pre-antral and small antral follicles up to $6 \mathrm{~mm}$ in diameter. Thus, level of AMH may represent the population of these follicles. It has been shown that the number of antral follicles in the ovaries is proportionally related to the size of primordial follicle stock from which they were recruited $^{(8)}$.

Antral follicle count (AFC) has the preferable achievement in predicting ovarian response to exogenous FSH. Ovarian antral follicles larger than $2 \mathrm{~mm}$ are extremely sensitive and responsive to FSH and are defined as "recruitable". They can be visualized and measured with transvaginal ultrasound and the total number of $2-10 \mathrm{~mm}$ follicles in both the ovaries represents the $\mathrm{AFC}{ }^{(9)}$.

Three-dimensional ultrasound also allows an objective appreciation of ovarian volume and total blood flow to the ovary, both of which have been suggested as important determinants of ovarian reserve. Further estimate is needed to define whether $3 \mathrm{D}$ measures of ovarian reserve show any clinical advantage over the clinical and endocrine markers of ovarian reserved ${ }^{(10)}$.

Ovarian follicular granulosa cells manufacture AMH and inhibin B. Both these hormones are produced by small antral follicles and could be useful endocrine markers of the antral follicle pool in the early follicular phase. Inhibin B levels in serum rise in the early follicular phase and peak around day's five to six of the cycle and then gradually falls in the late follicular phase and is almost below detection in the luteal phase with a small peak in levels post-ovulation ${ }^{(11)}$. However, many studies concluded that ESHRE's definition can't be popularized and the definition of ovarian response to ICSI should be determined for each population distinguished and should consider retrieved oocytes quality rather than quality ${ }^{(12)}$.

\section{PATIENTS AND METHODS:}

This study was conducted at Alexandria Armed Military Hospital from October 2016 to August 2018. A sample of 100 women aged between 25 and 35 years, was enrolled for intracytoplasmic sperm injection (ICSI) with the following inclusion criteria: First stimulation cycle for intracytoplasmic sperm injection (ICSI), a long protocol for the use of a gonadotrophin releasing hormone agonist (GnRHa), the presence of both ovaries, regular menstrual cycles, unexplained infertility, infertility due to fallopian tube factor, no evidence of endocrine disorders including, diabetes mellitus (normal TSH, prolactin), a body mass index ranging from 25 to $35 \mathrm{Kg} / \mathrm{m}^{2}$, not on hormone therapy as human chorionic gonadotropin (HCG), human menopausal gonadotropin (HMG) before ICSI cycle, no history of ovarian surgery, serological markers for hepatitis B, C, and HIV are negative. Also exclude women with previous trial for IVF, polycystic ovarian syndrome, any known pathology in ovaries, endometriosis, history of endocrine disorders as diabetes mellitus, hyperprolactinemia, patients who consume alcoholic beverages or smoke tobacco, patients with irregular cycle.

Before being recruited to the study, an informed consent document, in Arabic language, contains all locally required elements and specifies who informed the patient was written.

All women fulfilled a general information data_for each patient. Before any procedure, and after patient counseling, a written consent was obtained afterwards; all patients had a baseline hormonal profile (serum FSH, LH, $\mathrm{TSH}$, prolactin, E2,) on day 3 of non-treated cycle within 3 months before ovarian stimulation for ICSI. The study was approved by the Ethics Board of Al-Azhar University.

All women underwent suppression therapy using $\mathrm{GnRH}$ analogue long protocol starting from the preceding cycle day 21. Ovarian hyper-stimulation was subsequently started with HMG ampoules on day 3 of the following cycle according to the protocol applied.

Regular visits according to ovarian response were designed for each woman till reaching 3 follicles or more measuring $\geq 17 \mathrm{~mm}$. A date was then decided for HCG administration, after which a programmed plan for oocytes retrieval was then scheduled. Oocytes retrieval was carried out as planned followed by luteal phase support using natural progesterone supplements. Finally an assisted 
reproduction cycle data from (addendum II) for each patient was recorded. All participants had a transvaginal scan performed by a single investigator using a (TOSHIBA; Medical system, XARIO SSA-660, JAPAN) and a four-dimensional 5- to 9-MHz transvaginal probe. Participants were scanned in lithotomy position to limit discomfort and ensure free manipulation of the transvaginal transducer. Our technique briefly included:-

\section{Two-dimensional ultrasound:}

Two-dimensional ultrasound assessment of the pelvis to exclude any obvious pathology before the application of a region of interest over the ovary that defined the volume to be acquired. An automated mechanical sweep of this region through 90 was then undertaken using the slow sweep mode and the resultant multiplanar display examined to ensure that the entire ovary had been captured.

\section{Power Doppler:}

Power Doppler was then applied using predefined settings, which offer the best compromise between small ovarian vessel detection and artefact ${ }^{(13)}$. The volume mode was entered once an adequate power Doppler signal had been obtained and the resultant truncated sector defining the area of interest moved and adjusted to ensure that a complete ovarian volume was obtained. Two volume acquisitions for each ovary, one with gray scale and the other with power Doppler information, were obtained.

The data were subsequently transferred to a personal computer by a universal serial bus device without any data compression.

\section{D ultrasound:}

All measurements were made on a personal computer using 4D View (TOSHIBA; Medical system, XARIO SSA-660, JAPAN) by a single investigator. The $3 \mathrm{D}$ gray scale ovarian volume dataset was initially displayed in the multiplanar view and the total number of antral follicles measuring 2 $10 \mathrm{~mm}$ in diameter were counted as previously described (14). Briefly, this involved measurement of largest follicle in two planes to ensure this was $10 \mathrm{~mm}$ or smaller and counted all thereafter. All follicles measuring more than $10 \mathrm{~mm}$ were excluded. Virtual organ computer-aided analysis (VOCAL) was used to measure ovarian volume through the delineation of the ovarian cortex in the B (transverse image) plane as the volume was rotated 180 through 9 rotation steps ${ }^{(15)}$. Quantification of power Doppler information within the resultant 3D ovarian model was performed using "histogram facility" and three indices of vascularity were generated: the vascular index represents the ratio of power Doppler information within the total dataset relative to both color and gray information, the flow index reflects the mean power Doppler signal intensity, and the vascularization flow index, which is a combination of the other two indices ${ }^{(16)}$.

\section{Basal ovarian reserve screening:}

Serum E2 was measured in plasma specimens with an electrochemiluminescence immunoassay analyzer, using a commercial kit according to the manufacture's sensitized assay protocol. Serum FSH, LH were measured in plasma specimens with an electrochemiluminescence immunoassay (ECLIA) on the Roche Elecsys2010 immunoassay analyzer, using a commercial kit according to the manufacture's sensitized assay protocol. Also serum inhibin B hormone was measured in day 3 of the cycle prior to ART therapy using DSL-10-84100 an enzyme linked immunosorbant assay. A commercial ELISA kit (Diagnostic Systems Laboratories, Inc. Texas, USA) was used according to the manufacturer's protocol. The sensitivity of the assay is $7 \mathrm{pg} / \mathrm{ml}$ and AMH can be measured at any time in menstrual cycle as it is stable throughout the cycle. Serum was stored at $-20^{\circ} \mathrm{C}$ and assayed for $\mathrm{AMH}$. Serum and follicular-fluid AMH concentrations were determined using an ultrasensitive Enzyme-linked immunosorbent assay (ELISA) ${ }^{(17)}$.

The patients underwent ovarian stimulation following down-regulation with a GnRH agonist in a long protocol. Pituitary suppression with triptorelin acetate, 0.1 mg/day subcutaneously (Decapeptyl, Ferring Pharmaceuticals, Switzerland), was initiated 5-7 days before the estimated start of next menses and continued until the end of gonadotrophin administration. Ovarian stimulation is started after confirmation of downregulation when E2 levels are low. HMG started at a dose of 225-450 IU/day (dose is set based on patients age, BMI and past history) for the first 5 days and was followed by individual dose-adjustments 
according to the patient's follicular response as exclusively measured by transvaginal ultrasound (TVU). The daily dose could either be increased or decreased by 75 IU per adjustment, then monitoring of ovulation, based on response to the dose was adjusted. 10,000 IU urinary hCG (Choriomon, IBSA, Lugano, Switzerland) intramuscularly, was used to induce final follicular maturation when three or more follicles measuring $\geq 17$ $\mathrm{mm}$ in diameter were observed and was administered $36 \pm 2 \mathrm{~h}$ before planned oocyte retrieval.

\section{Oocytes Retrieval:}

All patients attended the Assisted Reproduction Unit for the planned oocytes retrieval. Patient was fasting to allow safe intubation. A venous indwelling cannula was placed for intravenous administration of fluids and medications. General anesthesia was used in all patients. Patient in lithotomy position and empty the urinary bladder. The vagina was washed by physiological saline. The transducer was connected to the ultrasound system. The direction of the guide beam was checked. The puncturing needle was connected to an aspiration/flushing apparatus attached by a fixation ring to the front and rear ends of the vaginal transducer, thereby defining the direction of puncture corresponding to the guide beam on the ultrasound image. The aspiration/washing apparatus was checked using test tubes. The transducer was dipped in $0.9 \%$ saline and cautiously introduced into the vagina. The uterus, both ovaries and the iliac vessels were identified by the visualization in both planes. The distance between the upper pole of the vagina and ovary was closely evaluated (care was taken to avoid vascular or intestinal interposition). Depth localization of the closest accessible follicle (distance from the upper vaginal pole to the center of the follicle) was done. Needle was pushed forcefully and carefully forward to the center of the follicle. Needle tip ridges were shown in the ultrasound image. While keeping the needle in place, the adjacent follicles were punctured without retracting the needle from the abdominal cavity between aspirations. The contents of the follicle were aspirated using an automatic suction apparatus. The aspirated follicular fluid was assessed (serous, blood tinged, bloody, blood). If necessary, the follicle was flushed. This may be repeated.
The tube with the follicular fluid was closed and passed on to the biologist. Ultrasound monitoring for any intraabdominal bleeding was done. Speculum was introduced to evaluate any bleeding from the posterior vaginal fornix. The patient was monitored for 2 hours (risk of bleeding). After aspiration of all accessible ovarian follicles, the follicular fluid was centrifuged to separate the cellular particle. Grading of oocyte was done. The oocytes were placed in culture medium and intracytoplasmic sperm injection was performed using Olympus CK40 inverted phase microscope and micromanipulating equipment. The injected oocytes were incubated at 37C. Fertilization was diagnosed by the presence of two proncuclei in the injected oocyte. Embryo quality was assessed according to presence of nuclear fragments. Embryo with $<10 \%$ nuclear fragments, with $10-20 \%$ fragments and with $>20 \%$ fragments was referred to as grade 1 , grade 2 and grade 3 respectively ${ }^{(18)}$.

As regards number of oocytes retrieved: High responders: those who respond to stimulation protocol with follicles No>20. Optimal responders: those who respond to stimulation protocol with follicle No: 5-20. Poor responders: those who respond to stimulation protocol with follicles No $<5$. This classification was based upon definitions of patients with poor ovarian response: follicles less than 5. While those with hyperstimulation ovarian response: follicles more than 30 per cycle ${ }^{(19)}$.

\section{Embryo transfer:}

It was done two to three days after oocytes retrieval, with no anesthesia in the lithotomy position. Up to three good quality embryos were transferred with a thin plastic cannula attached to a syringe (Cook embryo transfer catheter). The cervix was exposed with a sterile warm speculum and the external os was gently swabbed with culture medium after aspiration of cervical mucus by pipette. The position of the uterus was judged by the angle of the cervix to allow the catheter to be inserted in the appropriate direction.

Clinical outcomes of ICSI: The catheter was passed through the cervix to approximately 1 $\mathrm{cm}$ below the uterine fundus guided by ultrasound. Embryos were loaded in the distal end of the transfer catheter in culture fluid and were gently injected using a small tuberculin 
syringe. The patient rested for 30 minutes following the procedure before discharge.

The Hosmer-Lemeshow statistic evaluates the

goodness-of-fit by creating 10 ordered groups of subjects and then compares the number actually in each group (observed) to the number predicted by the logistic regression model (predicted. The receiver operating characteristic (ROC) curve define how accurately the deduced equation predicts the response and displays the full picture of trade-off between the sensitivity (true positive rate) and specificity (false positive rate) across a series of cut-off points. AUROC is considered as an effective measure of inherent validity of a predictive model. The larger the AUROC, the better is overall performance of the model to correctly predict the outcome for each subject Two tailed independent t-test were used to compare parametric variables. Wilcoxon rank sum test was used for skewed data to estimate p-value. Chi-square test with Yate's correction was used to compare proportions. All values considered statistically significant at $\mathrm{p}<0.05$.

\section{RESULTS:}

The mean age and mean FSH level of this group was $28.71 \pm 3.73$ years (25-35 years) and 13.31 $\pm 7.71 \mathrm{IU} / \mathrm{L}(4.87-45.08 \mathrm{IU} / \mathrm{L})$, respectively. Their mean BMI was $23.15 \pm$ $3.62 \mathrm{~kg} / \mathrm{m}^{2}\left(25-35.2 \mathrm{~kg} / \mathrm{m}^{2}\right)$. Basal serum AMH ng/ml $2.97 \pm 1.56$ (0.75-6.06), basal serum Inhibin B pg/ml $77 \pm 25.5$ (20-189) and $\mathrm{LH}(\mathrm{mIU} / \mathrm{ml}) 8.44 \pm 6.02$ (1.2-40.98). The participants had a variety of causative factors for their subfertility.

Outcomes of controlled ovarian stimulation (COS): No. of total oocyte retrieved $8.74 \pm$ 3.1(2-16), germinal vesicle (G V) $1.27 \pm 0.58$ (0-3) MI oocytes, $1.35 \pm 0.86$ (0-4), MII oocytes $4.93 \pm 2.24$ (0-11), Atretic oocytes $1.16 \pm 0.62(0-3)$, and fertilized oocyte $3.71 \pm$ $1.91(0-8)$. But the clinical outcomes of ICSI: Chemical pregnancy (No \%) positive 76 (76\%), negative 24 (24\%). Also clinical pregnancy (No \%) positive71 (71\%) negative29 (29\%) but cancellation rate (No $\%$ ) cancelled 4 (4\%) non-cancelled $96(96 \%)$ The degree of variability in baseline characteristics represented by coefficient of variation percentage (CV \%) which is $\mathrm{SD} /$ mean $100 \%$. CV\% shouldn't exceed $40 \%$ to confirm data homogeneity and controlled variation in subsequent statistical analyses. Variables having $\mathrm{CV} \%>40 \%$ were $\log$ transformed first before inclusion in any subsequent statistical analysis.

Overall 6 participants $(6 \%)$ demonstrated poor ovarian response; the mean age and mean FSH level of this group was $33.83 \pm 1.84$ years and $26.33 \pm 12.05 \mathrm{I} \mathrm{U} / \mathrm{L}$, respectively. Their mean BMI was $24.63 \pm 4.47 / \mathrm{m}^{2}$. Basal serum AMH ng/ml $1.68 \pm 1.05$ ), Basal serum Inhibin B pg/ml $25 \pm 5.6$ and $\mathrm{LH}(\mathrm{mIU} / \mathrm{ml})$ $17.15 \pm 11.69$.

Outcomes of controlled ovarian stimulation (COS): No among poor responders of total oocyte retrieved $2.50 \pm 0.55$. Germinal vesicle $(\mathrm{G} \mathrm{V}) 0.67 \pm 0.52 \mathrm{MI}$ oocytes, $0.83 \pm$ 0.41 , MII oocytes $0.5 \pm 0.55$, atretic oocytes $0.17 \pm 0.41$, and fertilized oocyte 0.5 \pm 0.55 . But the clinical outcomes of ICSI: Chemical pregnancy (No \%) positive 0 $(00 \%)$, negative $6(100 \%)$. Also clinical pregnancy $(\mathrm{n}, \%)$ positive $0(00 \%)$ negative 6 $(100 \%)$ but Cancellation rate (No \%) cancelled $0(00 \%)$ con-cancelled $6(100 \%)$ showed in table (1).

Overall 94 participants (94\%) demonstrated good ovarian response; the mean age and mean FSH level of this group was $28.38 \pm$ 3.58 years and $12.48 \pm 6.62 \mathrm{IU} / \mathrm{L}$, respectively. Their mean BMI was $26.25 \pm$ $3.57 / \mathrm{m}^{2}$. Basal serum AMH ng/ml $3.05 \pm$ 1.55 , basal serum inhibin B pg/ml $76 \pm 39.06$ and LH $(\mathrm{mIU} / \mathrm{ml}) 7.88 \pm 5.1$.

Outcomes of controlled ovarian stimulation (COS): No among poor responders of total oocyte retrieved $9.14 \pm 2.74$. Germinal vesicle $(\mathrm{G} \mathrm{V}) 1.31 \pm 0.57$. MI oocytes, $1.38 \pm$ 0.87 , MII oocytes $5.21 \pm 1.99$, atretic oocytes $1.22 \pm 0.57$, and fertilized oocyte 3.92 \pm 1.78 . But the clinical outcomes of ICSI: Chemical pregnancy (No \%) positive 76 (80.9\%), pegative $18(19.1 \%)$. Also clinical pregnancy (No \%) positive 71 (75.5\%), negative $23(24.5 \%)$ but cancellation rate (n\%) cancelled 4 (4.3\%) non-cancelled 90 (95.7\%) showed in table (1).

Table (1): comparison between poor responders and good responders group.

\begin{tabular}{||c|c|c|c||}
\hline Variable & $\begin{array}{c}\text { Poor } \\
\text { responder } \\
\mathbf{s} \\
(\mathbf{n}=6)\end{array}$ & $\begin{array}{c}\text { Good } \\
\text { responder } \\
\mathbf{s}(\mathbf{n}=94)\end{array}$ & P-value \\
\hline \hline \multicolumn{4}{|c||}{ Patient demographics } \\
\hline Age & $33.83 \pm$ & $28.38 \pm$ & $<0.001$ \\
\hline
\end{tabular}




\begin{tabular}{|c|c|c|c|}
\hline (Years) & 1.84 & 3.58 & (S) \\
\hline $\begin{array}{c}\mathrm{BMI} \\
\left(\mathrm{Kg} / \mathbf{m}^{2}\right)\end{array}$ & $\begin{array}{c}24.63 \pm \\
4.47\end{array}$ & $\begin{array}{c}26.25 \pm \\
3.57\end{array}$ & $\begin{array}{l}0.29 \\
\text { (NS) }\end{array}$ \\
\hline \multicolumn{4}{|c|}{ 3D/US findings } \\
\hline AFC & $\begin{array}{c}11.83 \pm \\
5.85\end{array}$ & $\begin{array}{c}17.99 \pm \\
4.86\end{array}$ & $0.004(\mathrm{~S})$ \\
\hline $\begin{array}{c}\text { Total } \\
\text { number of } \\
\text { follicles }\end{array}$ & $7.6 \pm 1.67$ & $\begin{array}{c}15.73 \pm \\
4.16\end{array}$ & $\begin{array}{l}<0.001 \\
(\mathrm{~S})\end{array}$ \\
\hline $\begin{array}{c}\text { Mean } \\
\text { ovarian } \\
\text { volume }\end{array}$ & $5.3 \pm 1.5$ & $2.5 \pm 9.9$ & $\begin{array}{c}>0.05(\mathrm{~N} \\
\mathrm{S})\end{array}$ \\
\hline $\begin{array}{c}\text { Mean } \\
\text { vascular } \\
\text { flow index }\end{array}$ & $2.5 \pm 1.6$ & $0.3-10.9$ & $\begin{array}{l}0.70 \\
\text { (NS) }\end{array}$ \\
\hline \multicolumn{4}{|c|}{ Biomarkers of ovarian reserve and response } \\
\hline $\begin{array}{c}\text { Basal } \\
\text { serum } \\
\text { AMH } \\
(\mathrm{ng} / \mathrm{ml})\end{array}$ & $\begin{array}{l}1.68 \pm \\
1.05\end{array}$ & $3.05 \pm 1.55$ & $0.036(\mathrm{~S})$ \\
\hline $\begin{array}{c}\text { Basal } \\
\text { serum } \\
\text { Inhibin B } \\
(\mathrm{pg} / \mathrm{ml})\end{array}$ & $25 \pm 5.6$ & $76 \pm 39.06$ & $0.005(\mathrm{~S})$ \\
\hline $\begin{array}{c}\text { Basal } \\
\text { serum FSH } \\
(\mathrm{mIU} / \mathrm{ml})\end{array}$ & $\begin{array}{l}26.33 \pm \\
12.05\end{array}$ & $\begin{array}{c}12.48 \pm \\
6.62\end{array}$ & $0.037(\mathrm{~S})$ \\
\hline $\begin{array}{c}\text { Basal } \\
\text { serum LH } \\
(\mathrm{mIU} / \mathrm{ml})\end{array}$ & $\begin{array}{c}17.15 \pm \\
11.69\end{array}$ & $7.88 \pm 5.1$ & $\begin{array}{l}0.11 \\
\text { (NS) }\end{array}$ \\
\hline \multicolumn{4}{|c|}{ Outcomes of COS } \\
\hline $\begin{array}{c}\text { Oocyte } \\
\text { retrieval }\end{array}$ & $\begin{array}{c}2.50 \pm \\
0.55 \\
\end{array}$ & $9.14 \pm 2.74$ & $\begin{array}{c}<0.001 \\
(\mathrm{~S}) \\
\end{array}$ \\
\hline $\begin{array}{c}\text { Germinal } \\
\text { vesicle (G } \\
\text { V) } \\
\end{array}$ & $\begin{array}{c}0.67 \pm \\
0.52\end{array}$ & $1.31 \pm 0.57$ & $0.027(\mathrm{~S})$ \\
\hline MI oocytes & $\begin{array}{c}0.83 \pm \\
0.41 \\
\end{array}$ & $1.38 \pm 0.87$ & $0.019(\mathrm{~S})$ \\
\hline $\begin{array}{c}\text { MII } \\
\text { oocytes }\end{array}$ & $0.5 \pm 0.55$ & $5.21 \pm 1.99$ & $\begin{array}{c}<0.001 \\
(\mathrm{~S})\end{array}$ \\
\hline $\begin{array}{l}\text { Atretic } \\
\text { oocytes } \\
\end{array}$ & $\begin{array}{c}0.17 \pm \\
0.41 \\
\end{array}$ & $1.22 \pm 0.57$ & $\begin{array}{c}<0.001 \\
(\mathrm{~S})\end{array}$ \\
\hline $\begin{array}{c}\text { Fertilized } \\
\text { oocyte }\end{array}$ & $0.5 \pm 0.55$ & $3.92 \pm 1.78$ & $\begin{array}{c}<0.001 \\
(\mathrm{~S}) \\
\end{array}$ \\
\hline \multicolumn{4}{|c|}{ Clinical outcomes of ICSI } \\
\hline $\begin{array}{c}\text { Chemical } \\
\text { pregnancy } \\
\text { (No\%) } \\
\text { Positive } \\
\text { Negative } \\
\end{array}$ & $\begin{array}{l}0(0 \%) 6 \\
(100 \%)\end{array}$ & $\begin{array}{c}76 \\
(80.9 \%) \\
18 \\
(19.1 \%)\end{array}$ & $\begin{array}{l}<0.001 \\
(\mathrm{~S})\end{array}$ \\
\hline $\begin{array}{c}\text { Clinical } \\
\text { pregnancy } \\
\text { (No \%) } \\
\text { Positive } \\
\text { Negative } \\
\end{array}$ & $\begin{array}{l}0(0 \%) 6 \\
(100 \%)\end{array}$ & $\begin{array}{c}71 \\
(75.5 \%) \\
23 \\
(24.5 \%)\end{array}$ & $\begin{array}{l}<0.001 \\
(\mathrm{~S})\end{array}$ \\
\hline $\begin{array}{c}\text { Cancellatio } \\
\text { n rate (No } \\
\%) \\
\text { Cancelled } \\
\text { Non- } \\
\text { cancelled } \\
\end{array}$ & $\begin{array}{l}0(0 \%) 6 \\
(100 \%)\end{array}$ & $\begin{array}{c}4(4.3 \%) \\
90 \\
(95.7 \%)\end{array}$ & 1 (NS) \\
\hline
\end{tabular}

Frequency analysis of number of oocytes retrieved of the whole study groups $(\mathrm{No}=100)$, the 10 th percentile corresponds to the cut-off number of oocytes retrieved. To our case, 5 oocytes is considered a good response and patients with less than 5 oocytes retrieved are considered poor responders figure (1).

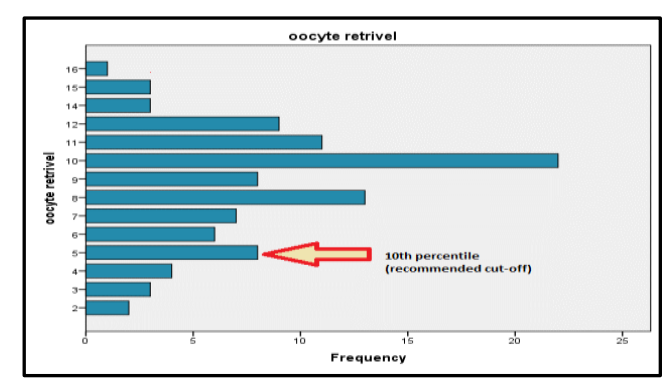

Figure (1) Frequency analysis of number of oocytes retrieved of the whole study groups

Frequency analysis of number of mature oocytes retrieved of the whole study groups $(\mathrm{No}=100)$, the 10th percentile corresponds to the cut-off number of mature oocytes retrieved. To our case, 2 oocytes is considered a good response and patients with less than mature oocytes retrieved are considered poor responders figure (2).

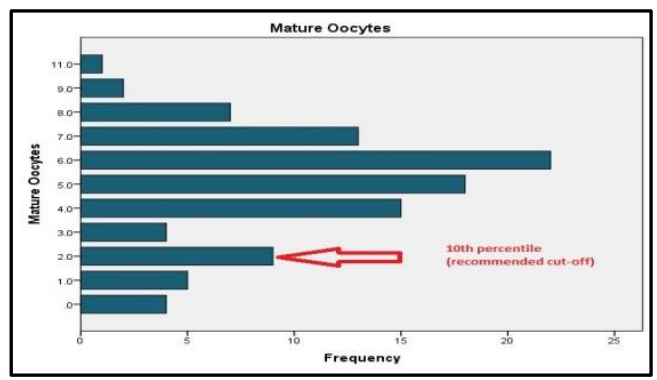

Figure (2) Frequency analysis of number of mature oocytes retrieved of the whole study groups

\section{ICSI Outcome analysis:}

\section{Assessment of oocytes quality:}

Comparing the mean no. of good \& bad oocytes in the study groups

\begin{tabular}{|l|c|c|c|}
\hline Variable & $\begin{array}{c}\text { Poor } \\
\text { responders } \\
\text { (n=6) }\end{array}$ & $\begin{array}{c}\text { Good } \\
\text { responders } \\
\text { (n=94) }\end{array}$ & $\begin{array}{c}\text { P } \\
\text { value }\end{array}$ \\
\hline \hline $\begin{array}{l}\text { Bad oocytes } \\
\text { (G1+G2) }\end{array}$ & $1.5 \pm 0.8$ & $2.26 \pm 1.14$ & $\begin{array}{c}0.013 \\
(\mathrm{~S})\end{array}$ \\
\hline $\begin{array}{l}\text { Good } \\
\text { oocytes } \\
\text { (G3+G4) }\end{array}$ & $0.67 \pm 0.52$ & $6.43 \pm 2.12$ & $\begin{array}{c}<.001 \\
(\mathrm{~S})\end{array}$ \\
\hline
\end{tabular}

\section{Effect of individual predictors of ovarian}

\section{response:}

Effect of antral follicular count:

Box plot of comparing the antral follicular count (AFC) in poor response and good response group. The bolded line inside each box represents the median value of AFC in each group. The plot demonstrates clearly that the AFC in poor response group is significantly higher than good response group Figure 
(3).

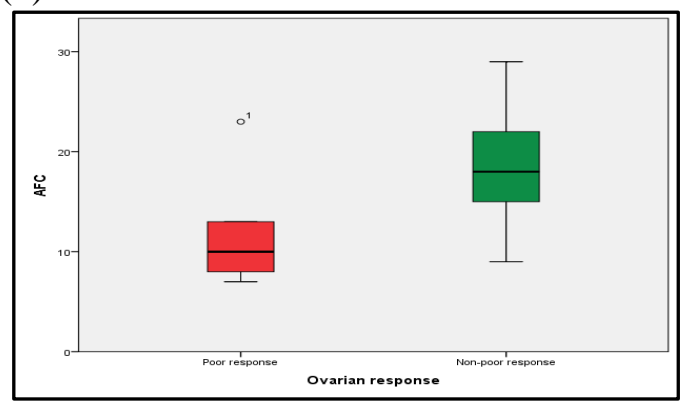

Figure (3) antral follicular count effect

Effect of anti-Mullerian hormone level:

Box plot of comparing the anti-Mullerian hormone (AMH) level in poor response and good response group. The bolded line inside each box represents the median value of $\mathrm{AMH}$ in each group. The plot demonstrates clearly that the AMH in poor response group is significantly less than good response group Figure (4).

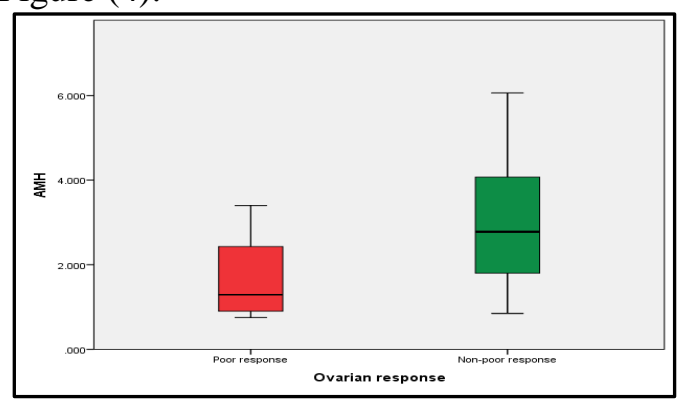

Figure (4) anti-Mullerian hormone level effect

Effect of Inhibin B hormone level:

Box plot of comparing the inihibin B level in poor response and good response group. The bolded line inside each box represents the median value of inhibin $B$ in each group. The plot demonstrates clearly that the inhibin B in poor response group is significantly less than good response group Figure (5)

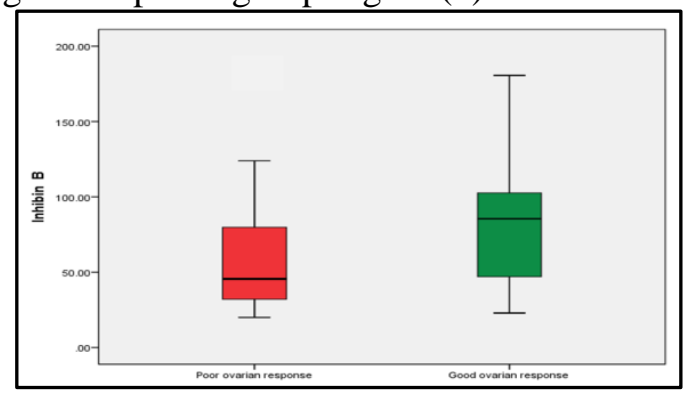

Figure (5) Inhibin B hormone level effect

This study revealed that there was significant difference between the age among good and poor responders, there was also very significant difference between good responders and poor responders in $\mathrm{AFC}$, AMH FSH, LH, E2, inhibin B. The good- response group had significantly higher chemical and clinical pregnancy rates.

\section{DISCUSSION:}

The aim of the present study is to compare the value of anti-Mullerian hormone (AMH), Inhibin-B and Three dimensional ultrasound parameters as markers for ovarian response in patients undergoing intracytoplasmic sperm injection cycles.

The mean AFC was, 17 ranged from 7 to 29 follicles, the mean AMH level was $2.97 \mathrm{ng} / \mathrm{ml}$, ranged from 0.75 to $6.06 \mathrm{ng} / \mathrm{ml}$ and the mean inhibin B level was $77.05 \mathrm{pg} / \mathrm{ml}$ and ranged from 20 to $189 \mathrm{pg} / \mathrm{ml}$.

In the present study, there is a significant difference between the age among good and poor responders and there was a significant difference between good responders and poor responders in $\mathrm{AFC}, \mathrm{AMH}$, inhibin $\mathrm{B}^{(20)}$.

It was found that there is variety in the role of FSH. In our study, FSH was establishing to be a perfect predictor of ovarian reserve. While in Alan study 2016 found that FSH has no role in prediction of ovarian reserve (21).

In the present study there was correlation between $\mathrm{AFC}, \mathrm{AMH}$, inhibin $\mathrm{B}$, and number of oocytes available for fertilization and these results also went with the results of Gutam study as there was good correlation between AFC, AMH, Inhibin B and number of oocytes available for fertilization. The potential value of AMH in predicting the probability of pregnancy after assisted conception was developed ${ }^{(22)}$. However, the accuracy curves in the present study indicate that AMH is a strong predictor for ovarian response but not for clinical pregnancy or live birth.

In the past, some authors contracted the view that AMH is associated with oocyte quality, but more subsequent studies explained that no such correlation exists ${ }^{(23)}$.

A recent powerful meta-analysis concluded that AMH alone may have some association with predicting live birth after IVF and may be helpful when counseling couples before undergoing fertility treatment, the availability of more embryos for transfer in patients with higher AMH rather than a direct association between AMH and embryo quality (24). 
Although ovarian volume was predictive of both the number of oocytes retrieved and poor response on univariate analysis, as reported in other studies, its predictive capacity was less than that of AFC and AMH. The superior predictive power of AFC above ovarian volume in this study is in convention with the majority of studies and a recent meta-analysis (25).

Ovarian vascularity, the other 3D ultrasound ovarian parameter studied, was not predictive of ovarian response in both univariate and multivariate analysis and was less predictive of the number of oocytes. This is in convention with all of the reported comparative studies that have used 3D ultrasound for blood flow assessment ${ }^{(26)}$.

The AFC assessment is operator dependent but this can be amended through the acquisition of 3D data and the subsequent offline assessment of these data, which has been shown to improve measurement accuracy even among unskilled observers. This does not validate the routine use of 3D ultrasound in clinical practice, and skilled observers can use real-time two-dimensional ultrasound to reliably determine the total AFC. The further ultrasound parameters derivable through $3 \mathrm{D}$ imaging, namely ovarian volume and vascularity, were not significant predictors in the multivariate analysis ${ }^{(27)}$.

\section{CONCLUSION:}

In conclusion, $\mathrm{AFC}, \mathrm{AMH}$ and inhibin $\mathrm{B}$ are effective in predicting the ovarian reserve as well as the response to induction, and both $\mathrm{AMH}$ and AFC are accurate for the assessment of ovarian reserve.

Inhibin B correlated moderately positively with AFC and number of collected oocytes also with the number of fertilized oocytes, also there is statistically significant correlation between inhibin $B$ and the fertilization rate. 3D ultrasonographic ovarian parameters, such as ovarian volume, vascularity, and perfusion, do not appear to have any significant value in the prediction of ovarian response.

\section{RECOMMENDATIONS:}

Based on our study findings, a list of recommendation should be taken into account to guide the future research and improve the clinical practice of ICSI.

For clinical practice:

The final deduced equation for prediction of poor ovarian response should guide the eligibility of women who are candidates for ICSI prior to implementation of therapeutic interventions. This will provide higher success rates and reduce both the total cost of treatment and cancellation rate, for better and faster implementation in clinical practice, the results of the current study should be framed into a program with simple graphical user interface or as nomogram. These practical tools can be carried out by a statistical modeler. Instead of the commonly used Bologna criteria, the adapted definition of poor ovarian response and the recommended new cut-off values of ovarian reserve tests should guide the ICSI practice in Egypt; as they are much more realistic and intrinsic to our patient population. Due to substantial variability observed throughout our study, we recommend the AMH assay to be standardized among the different laboratories to avoid the possible misclassification errors that may originate through the current assay methods.

For future research:

Similar studies including patients with past medical history significant to endocrine, gynecologic or obstetric disorders along with those having past-surgical history should be conducted to guide the clinical decision in such cases. The differential effects of different pre-ICSI protocols should be compared statistically, this will help to precisely choose the optimum protocol and optimize the doses on individual basis which result in higher success rates, a large-scale study including only the cases who had cycle cancellation should be carefully analyzed for better understanding of the etiology of cancellation and similar prediction equation should be deduced to predict the odds of cycle cancellation, The phenomena of ovarian hyperstimulation: definition, predictors and prognosis should be closely investigated through a patient oriented and individualized view. Through the current study, we evaluated the prediction of ovarian response 
under standard long-term protocol. Future studies should be conducted under similar methodology for different treatment protocols to investigate for inter-protocol variation and the differential safety and efficacy of the utilized medications which may guide the process of protocol selection on individual basis.

\section{REFERENCES:}

1. Chambers GM, Paul RC, Harris K, Fitzgerald $O$ and Boothroyd CV (2017): Assisted reproductive technology in Australia and New Zealand: cumulative live birth rates as measures of success., 207: 114-18.

2. Brockmans FJ, Looman C W, Habbema J D and Velde E (2004): Impact of repeated antral follicle counts on the prediction of poor ovarian response in women undergoing in vitro fertilization., 81:35-41.

3. Ferraretti. (2011): ESHRE consensus on the definition of "poor response" to ovarian stimulation for in vitro fertilization: 26:1616-24.

4. Iliodromiti S, Anderson RA and Nelson SM (2015). Technical and performance characteristics of anti-mullerian hormone and antral follicle count as biomarkers of ovarian response., 21:698-710. 5. Chow G E, Criniti A R and Soules M R (2004): AFC and serum FSH levels to asses functional ovarian aging., 104:801- 4.

6. Islam Y (2016): The value of different ovarian reserve tests in the prediction of ovarian response in patients with unexplained infertility 21(2):69-74.

7. Ragione DT, Verheyen G, Papanikolaou EG, Van Landuyt L, Devroey P, Van and Steirteghem A (2007): Developmental stage on day-5 and fragmentation rate on day- 3 can influence the implantation potential of top-quality blastocysts in IVF cycles with single embryo., 5(1):2.

8. Kelsey TW, Anderson RA, Wright P, Nelson SM and Wallace WH (2012): assessment of the human ovarian reserve., 18: 79-87.

9. Tan, $\mathbf{R}$ (2011): Comparisons of inhibin $\mathrm{B}$ versus antimüllerian hormone in poor ovarian responders undergoing in vitro fertilization, 96 (4): 905-11.

10. Ebner T, Sommergruber M, Moser M, Shebl O, Schreier-Lechner E and Tews
G (2006): Basal level of anti-Mullerian hormone is associated with oocyte quality in stimulated cycles, 21(8): 2022-26.

11. Van Montfoort APA, Dumoulin JCM, Land JA, Coonen E, Derhaag JG, and Evers JLH (2005): Elective single embryo transfer (eSET) policy in the first three IVF/ICSI treatment cycles, 20 (2): 433 36.

12. Wu MH, Pan HA and Chang FM (2017): Three-dimensional and power Doppler ultrasonography in infertility and reproductive endocrinology, 46:209.

13. Van Gorp $T$, Veldman $J$ and Calster B (2012): Subjective assessment by ultrasound is superior to the risk of malignancy index (RMI) or the risk of ovarian malignancy algorithm (ROMA) in discriminating benign from malignant adnexal masses, 48:1649-56.

14. Choh Y, Criniti A R and Soules $M$ R (2017): AFC and serum FSH levels to asses functional ovarian aging, 104:801-4.

15. Hendriks DJ, Kwee J, Mol B and Broekmans FJ (2007): Ultrasonography as a tool for the prediction of outcome in IVF patients, 87:764 -75.

16. Kanakaraj A, Shaik R, Farid J and Roshn R (2016): Comparative study of ovarian reserve in users and non-users of oral contraceptives, 15 (4): 69-72.

17. Van Rooij IA, Broekmans FJ, Velde ER, Fauser BC, Bancsi LF, Jong FH and Themmen AP (2002): Serum antiMullerian hormone levels: a novel measure of ovarian reserve, 17(12): 3065-71.

18. Amato $P$ (2017): Ovarian reserve testing, clinical reproductive medicine and surgery, 14: 259-68.

19. Pratap $K$ and Alok $S$ (2014): Bioactive FSH and inhibin concentrations during ovulation induction in patients with polycystic ovarian disease, 15:123.

20. Van Voorhis BJ (2007): Clinical practice in vitro fertilization, 356 (4): 379-86. 21. Alan $S$ and McNeilly (2016): Diagnostic applications for FSH, inhibin and activins molecular and cellular endocrinology, 359 : 121-25.

22. Gutam N, Rita BD, Rubina $M$ and Bruno L (2004): Stimulation strategies for complex IVF., 20 (2): 55-62. 
23. Hendriks DJ, Broekmans FJ and Bancsi LF (2005): Repeated cloniphene citrate challenge testing in the prediction of outcome in IVF and comparison with basal markers for ovarian reserve, 20 (1): 160- 63.

24. Panchal $S$ and Nagori CB (2009): Pre-hCG 3D and 3D power Doppler assessment of the follicle for improving pregnancy rates in intrauterine insemination cycles, 2:62-7.

25. Yates A, Roberts $S$ and Nardo L(2012): Anti-Mullerian hormone-tailored stimulation protocols improve outcomes whilst reducing adverse effects and costs of IVF., 27(2) :629-35.

26. Deekshya Shrestha, Xiaolin La and Huai L (2015): Comparison of different stimulation protocols used in in vitro fertilization, 3: 130- 37.

27. Fishel S, Aslam I, Lisi F, Rinaldi L, Timson J, Jacobson M, Gobetz L, Green S, Campbell A and Lisi R (2000): ICSI is the treatment of choice for all cases of in-vitro conception, 15(6):1278- 83 